\title{
Teaching NeuroImages: Slowly progressive hypertrophic brachial plexopathy due to SEPT9 mutation
}

Paulo Victor Sgobbi de Souza, MD, Eduardo Augusto Gonçalves, MD, Bruno de Mattos Lombardi Badia, MD, Igor Braga Farias, MD, Luiz Henrique Libardi Silva, MD, Mario Teruo Yanagiura, MD,

Wladimir Bocca Vieira de Rezende Pinto, MD, MSc, and Acary Souza Bulle Oliveira, MD, PhD

Neurology ${ }^{\circledR}$ 2020;95:e109-e110. doi:10.1212/WNL.0000000000009739

A 51-year-old man had slowly progressive muscle wasting, weakness, and paresthesia of the right upper limb since age 21 years. His deceased mother had a single episode of painful right upper limb paresis. Examination showed severe amyotrophy and hypoesthesia of the right upper limb. Neuroimaging studies disclosed marked involvement of the right brachial plexus (figures 1 and 2). Neurophysiologic studies disclosed severe right brachial plexopathy. Gene panel for inherited neuropathies disclosed pathogenic variant c.278C $>\mathrm{T}$ (p.Ser93Phe) in the SEPT9 gene, ${ }^{1,2}$ confirming the diagnosis of hereditary neuralgic amyotrophy. ${ }^{1,2}$ SEPT9-related disorders can present as autosomal dominant recurrent or progressive hypertrophic brachial plexus neuropathy. ${ }^{1,2}$

\section{Acknowledgment}

Full consent was obtained from the patient for the case report. This study was approved by our institutional ethics committee (CEP-UNIFESP/HSP).

\section{Study funding}

No target funding reported.

Figure 1 Examination in SEPT9-related brachial plexus neuropathy

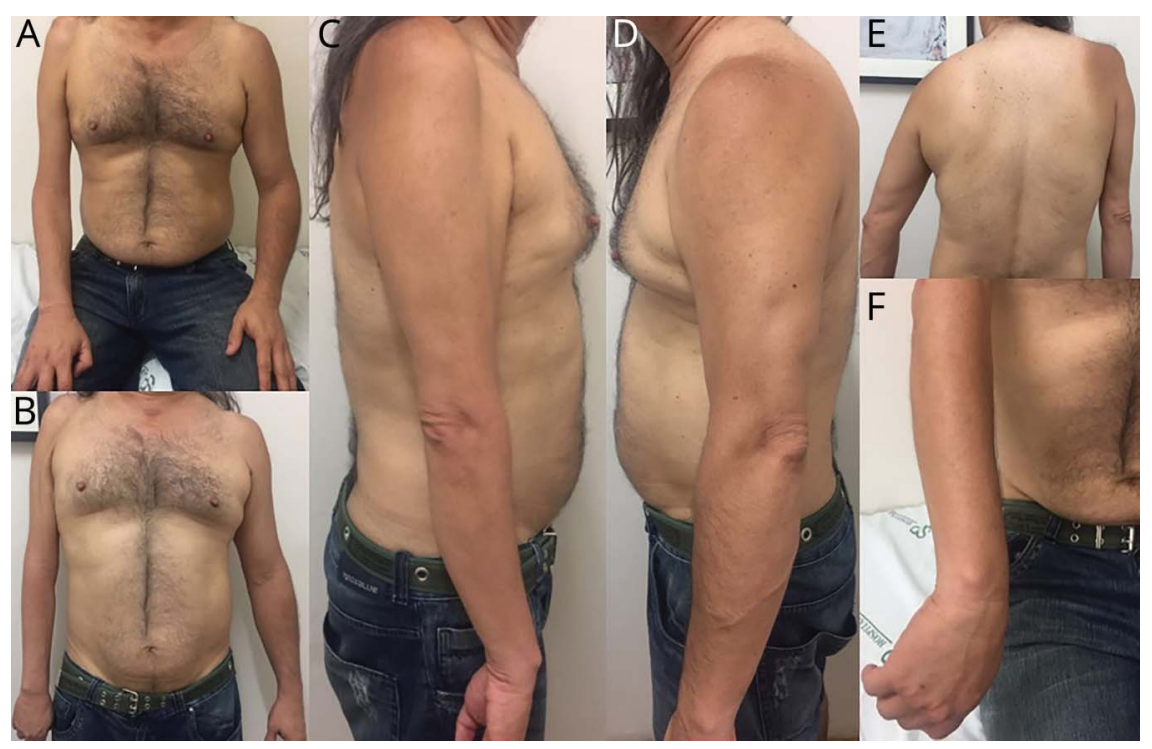

Evaluation shows marked amyotrophy of the right upper limb (A and B), mainly in the scapular girdle (C-E), arm, and (F) minor extent in the forearm and hand.

\section{Correspondence}

Dr. Pinto

wladimirbvrpinto@

gmail.com

\section{MORE ONLINE}

$\rightarrow$ Teaching slides

links.lww.com/WNL/

B101 
Figure 2 Muscle and brachial plexus imaging studies

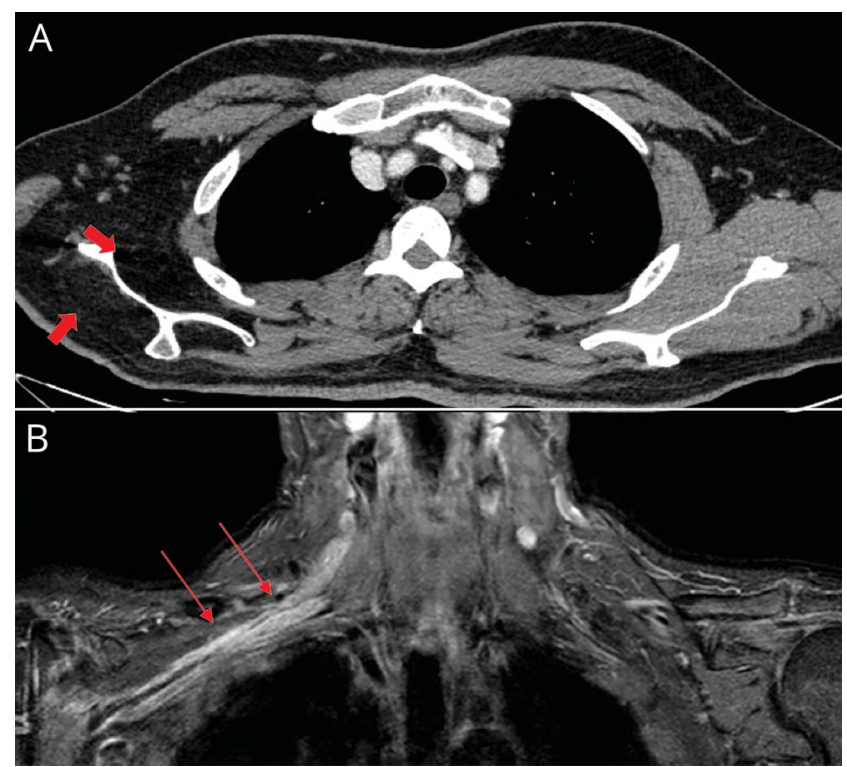

(A) Chest CT scan shows marked global amyotrophy and liposubstitution (wide red arrows) of the right scapular girdle muscle groups. (B) Coronal MRI of the brachial plexus shows asymmetric hyperintense signal and hypertrophy of right brachial plexus anterior roots, trunks, and cords in short tau inversion recovery sequence (red arrows).

\section{Disclosure}

The authors report no relevant disclosures. Go to Neurology. org/ $\mathrm{N}$ for full disclosures.

\section{Appendix Authors}

\begin{tabular}{lll}
\hline Name & Location & Contribution \\
\hline $\begin{array}{l}\text { Paulo Victor } \\
\text { Sgobbi de Souza, }\end{array}$ & $\begin{array}{l}\text { Federal University } \\
\text { of São Paulo } \\
\text { (UNIFESP), Brazil }\end{array}$ & $\begin{array}{l}\text { Case report project } \\
\text { (conception, organization, } \\
\text { execution), writing of the first } \\
\text { draft, review and critique }\end{array}$ \\
\hline
\end{tabular}

Appendix (continued)

\begin{tabular}{|c|c|c|}
\hline Name & Location & Contribution \\
\hline $\begin{array}{l}\text { Eduardo Augusto } \\
\text { Gonçalves, MD }\end{array}$ & $\begin{array}{l}\text { Federal University } \\
\text { of São Paulo } \\
\text { (UNIFESP), Brazil }\end{array}$ & $\begin{array}{l}\text { Case report project } \\
\text { (conception, organization, } \\
\text { execution), writing of the } \\
\text { first draft, review and } \\
\text { critique }\end{array}$ \\
\hline $\begin{array}{l}\text { Bruno de Mattos } \\
\text { Lombardi Badia, } \\
\text { MD }\end{array}$ & $\begin{array}{l}\text { Federal University } \\
\text { of São Paulo } \\
\text { (UNIFESP), Brazil }\end{array}$ & $\begin{array}{l}\text { Case report project } \\
\text { (conception, organization, } \\
\text { execution), writing of the } \\
\text { first draft, review and } \\
\text { critique }\end{array}$ \\
\hline $\begin{array}{l}\text { Igor Braga Farias, } \\
\text { MD }\end{array}$ & $\begin{array}{l}\text { Federal University } \\
\text { of São Paulo } \\
\text { (UNIFESP), Brazil }\end{array}$ & $\begin{array}{l}\text { Case report project } \\
\text { (conception, organization, } \\
\text { execution), writing of the } \\
\text { first draft, review and } \\
\text { critique }\end{array}$ \\
\hline $\begin{array}{l}\text { Luiz Henrique } \\
\text { Libardi Silva, MD }\end{array}$ & $\begin{array}{l}\text { Federal University } \\
\text { of São Paulo } \\
\text { (UNIFESP), Brazil }\end{array}$ & $\begin{array}{l}\text { Case report project } \\
\text { (conception, organization, } \\
\text { execution), writing of the } \\
\text { first draft, review and } \\
\text { critique }\end{array}$ \\
\hline $\begin{array}{l}\text { Mario Teruo } \\
\text { Yanagiura, MD }\end{array}$ & $\begin{array}{l}\text { Federal University } \\
\text { of São Paulo } \\
\text { (UNIFESP), Brazil }\end{array}$ & $\begin{array}{l}\text { Case report project } \\
\text { (conception, organization, } \\
\text { execution), writing of the } \\
\text { first draft, review and } \\
\text { critique }\end{array}$ \\
\hline $\begin{array}{l}\text { Wladimir Bocca } \\
\text { Vieira de Rezende } \\
\text { Pinto, MD, MSc }\end{array}$ & $\begin{array}{l}\text { Federal University } \\
\text { of São Paulo } \\
\text { (UNIFESP), Brazil }\end{array}$ & $\begin{array}{l}\text { Case report project } \\
\text { (conception, organization, } \\
\text { execution), writing of the } \\
\text { first draft, review and } \\
\text { critique }\end{array}$ \\
\hline $\begin{array}{l}\text { Acary Souza Bulle } \\
\text { Oliveira, MD, PhD }\end{array}$ & $\begin{array}{l}\text { Federal University } \\
\text { of São Paulo } \\
\text { (UNIFESP), Brazil }\end{array}$ & $\begin{array}{l}\text { Case report project } \\
\text { (conception), review and } \\
\text { critique }\end{array}$ \\
\hline
\end{tabular}

\section{References}

1. Chance PF. Inherited focal, episodic neuropathies: hereditary neuropathy with liability to pressure palsies and hereditary neuralgic amyotrophy. Neuromolecular Med 2006;8:159-174.

2. Hannibal MC, Ruzzo EK, Miller LR, et al. SEPT9 gene sequencing analysis reveals recurrent mutations in hereditary neuralgic amyotrophy. Neurology 2009;72: 1755-1759. 


\section{Neurology}

Teaching NeuroImages: Slowly progressive hypertrophic brachial plexopathy due to SEPT9 mutation

Paulo Victor Sgobbi de Souza, Eduardo Augusto Gonçalves, Bruno de Mattos Lombardi Badia, et al.

Neurology 2020;95;e109-e110 Published Online before print June 10, 2020

DOI 10.1212/WNL.0000000000009739

This information is current as of June 10, 2020

Updated Information \& Services

References

Subspecialty Collections

Permissions \& Licensing

Reprints including high resolution figures, can be found at: http://n.neurology.org/content/95/1/e109.full

This article cites 2 articles, 1 of which you can access for free at: http://n.neurology.org/content/95/1/e109.full\#ref-list-1

This article, along with others on similar topics, appears in the following collection(s):

Anterior nerve cell disease

http://n.neurology.org/cgi/collection/anterior_nerve_cell_disease MRI

http://n.neurology.org/cgi/collection/mri

Peripheral neuropathy

http://n.neurology.org/cgi/collection/peripheral_neuropathy

Information about reproducing this article in parts (figures,tables) or in its entirety can be found online at:

http://www.neurology.org/about/about_the_journal\#permissions

Information about ordering reprints can be found online:

http://n.neurology.org/subscribers/advertise

Neurology ${ }^{\circledR}$ is the official journal of the American Academy of Neurology. Published continuously since 1951, it is now a weekly with 48 issues per year. Copyright () 2020 American Academy of Neurology. All rights reserved. Print ISSN: 0028-3878. Online ISSN: 1526-632X.

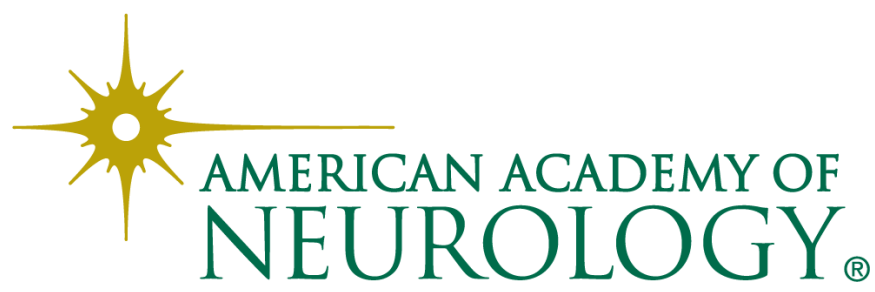

